\title{
BMJ Open Comprehensive geriatric assessment in perioperative care: a protocol for a systematic review and qualitative synthesis
}

\author{
Rachael Lucia Miller (D) ,, Jonathan David Barnes, ${ }^{3}$ Ronelle Mouton, ${ }^{3}$ \\ Philip Braude, ${ }^{4}$ Robert Hinchliffe $e^{1,5}$
}

To cite: Miller RL, Barnes JD, Mouton $\mathrm{R}$, et al. Comprehensive geriatric assessment in perioperative care: a protocol for a systematic review and qualitative synthesis. BMJ Open 2021;11:e049875. doi:10.1136/ bmjopen-2021-049875

- Prepublication history and additional supplemental material for this paper are available online. To view these files, please visit the journal online (http://dx.doi.org/10.1136/ bmjopen-2021-049875)

Received 03 February 2021 Accepted 11 November 2021

Check for updates

(C) Author(s) (or their employer(s)) 2021. Re-use permitted under CC BY. Published by BMJ.

${ }^{1}$ Translational Health Sciences, University of Bristol, Bristol, UK ${ }^{2}$ Vascular Surgery, Musgrove Park Hospital, Taunton, UK

${ }^{3}$ Anaesthesia, North Bristol NHS Trust, Bristol, UK

${ }^{4}$ CLARITY (Collaborative Ageing Research) group, Department of Medicine for Older People, North Bristol NHS Trust, Bristol, UK

${ }^{5}$ Vascular Surgery, North Bristol NHS Trust, Bristol, UK

Correspondence to Ms Rachael Lucia Miller; rm17210@bristol.ac.uk

\section{ABSTRACT}

Introduction Comprehensive geriatric assessment (CGA) is an intervention that has been deployed in the perioperative setting with the aim to improve outcomes for older patients admitted to hospital. Older patients undergoing surgery are more likely to have postoperative complications, a longer hospital stay and be discharged to a care facility. Despite the increasing application of this intervention within surgical services, the evidence for CGA remains limited in this group. The aim of this systematic review is to describe CGA as in intervention applied to surgical populations in randomised controlled trials (RCTs) as well as the outcomes assessed.

Methods and analysis A systematic search of RCTs of CGA in surgery will be run in Embase, Medline, CINAHL (Cumulative Index to Nursing and Allied Health Literature) and Cochrane library. Further articles will be identified from reference lists in relevant studies found in the search. A narrative synthesis will be undertaken outlining specialties included, detailed descriptions of the intervention and outcomes.

Ethics and dissemination No ethical approval is required. The results of this review will be published and used as the basis of work to optimise this intervention for future trials in surgical populations.

PROSPERO registration number This review is registered with PROSPERO CRD42020221797.

\section{INTRODUCTION \\ Rationale}

The average age of surgical patients is increasing bringing novel challenges to healthcare professionals within the perioperative pathway. ${ }^{12}$ Compared with younger patients, older people have a higher postoperative mortality and are more likely to experience significant postoperative complications, longer length of hospital stay and greater likelihood of discharge to a care facility. ${ }^{3}$ For example, according to the latest report from the National Emergency Laparotomy Audit, the 30-day mortality in patients over 65 years old and living with frailty was considerably
Strengths and limitations of this study

- This will be the first methodological, systematic review to conduct a qualitative analysis and summarise the reporting of comprehensive geriatric assessment (CGA) as an intervention in perioperative care.

- Only randomised controlled trials evaluating CGA in the perioperative period as an intervention will be included.

- This review will not report a meta-analysis of quantitative results.

- This review will describe how trials report CGA standard of care, a novel aspect.

above average at $18 \%$ compared with the overall $9.3 \%$ for this surgery. ${ }^{1}$

Comprehensive geriatric assessment (CGA) has been employed to improve outcomes for older patients admitted to hospital. Originally described in the 1930 s, descriptions and practice of CGA have varied widely in the literature. ${ }^{4}$ CGA is frequently defined as a 'multidimensional diagnostic and therapeutic process that is focused on determining a frail older person's medical, functional, mental, and social capabilities and limitations with the goal of ensuring that problems are identified, quantified, and managed appropriately'. ${ }^{5}$ It has been widely adopted in the care of the hospitalised older person, with an associated reduction in 1-year mortality and institutionalisation posthospital discharge.$^{5}$ Evidence of benefit within surgical populations is more limited and have focused mainly on patients who need surgery for hip fracture. ${ }^{6}$ The most recent Cochrane review on perioperative CGA lacked generalisability to all surgical disciplines due to the limited populations the randomised trials included: seven trials in hip fracture, and one in elective surgical oncology. ${ }^{6}$ Since the search was conducted in 
January 2017 further trials have been completed in other surgical specialties. While the Cochrane review focused on the health outcomes of CGA in a perioperative setting, this protocol describes a systematic review that will develop the existing knowledge by focussing on qualitative analysis of the literature, paying particular attention to the timing, components and team members involved in the intervention.

There is currently significant variation in how CGA is defined and reported in clinical research with no robustly developed consensus definitions. ${ }^{7-9}$ Definitions of perioperative CGA vary from which multidisciplinary team members should be included, which domains should be assessed and optimised, when is the right point of delivery (preoperatively or postoperatively) and even which patients should be selected. ${ }^{8}$ This provides a lack of standardisation in delivery of CGA and which aspects could be strengthened, or removed, to increase the efficacy of this complex intervention to achieve positive outcomes. ${ }^{10}$ One recent review has attempted to outline the core components of CGA in medical patients. ${ }^{8}$ However, no study has fully laid out the features of trial design or analysed the variation of delivery of this intervention for surgical patients. ${ }^{1}$

This protocol is designed to systematically review and summarise the reporting of CGA as an intervention in perioperative randomised controlled trials (RCTs). It will be reported according to Preferred Reporting Items for Systematic Review and Meta-Analysis Protocols statement (online supplemental information 1). ${ }^{11}$

\section{Aim}

The aim of this systematic review is to describe CGA as in intervention applied to surgical populations in RCTs.

\section{Specific objectives}

1. Examine the described components of CGA as an intervention in identified trials, including how, when and by whom these are delivered.

2. Identify surgical populations where randomised controlled studies have been performed comparing CGA to any other care, in either an elective or emergency surgical population.

3. Describe how trials report 'standard care'.

4. Determine what outcomes have been used to assess effectiveness of CGA and whether these reflect a biological plausibility of how CGA affects outcomes

\section{Methods}

Data item numbers collected include:

1. Participants: sex, age, number randomised, target sample size, reasons for non-recruitment, surgical specialty, emergency/elective population.

2. Interventions: description of interventions including: components of CGA, healthcare professional delivering intervention, assessment/management tools used (if relevant), time point delivered, duration of time spent with patient, detail of assessment made, detail of care delivered, setting of intervention (eg, clinic, separate ward).

3. Standard care: comparator description, healthcare professionals delivering care in comparator/control group.

4. Outcomes: list of reported outcomes, quantitative data for 11 key areas as defined by Core Outcome Measures in Perioperative and Anaesthetic Care-standard endpoints for perioperative medicine (COMPAC-StEP) working group where possible, including patient comfort, clinical indicators, cognition and stroke, cardiovascular, respiratory, renal, bleeding, morbidity, survival, patient centred outcomes and healthcare resource utilisation. ${ }^{12}$

\section{Data sources and search strategy}

A search strategy was adapted from a previous Cochrane review. ${ }^{6}$ It includes the themes 'geriatric care,' 'frailty,' 'surgery or trauma,' 'randomised controlled trials.' This will be performed across EMBASE, Medline, CINAHL and Cochrane library with help from an information specialist (online supplemental information 2).

\section{Study selection, inclusion and exclusion criteria}

Any RCT of CGA versus a control group (standard care) will be included. There will be no age cut-off for the purpose of this review, so that it can identify who has received the intervention, although it is anticipated that studies will include patients 60 years and over.

For the purpose of inclusion, if not otherwise identified as CGA, this study will define perioperative CGA as any review of a patient in the perioperative period by a healthcare professional with training in geriatric medicine (eg, consultant, trainee, specialist nurse). Review exclusively by any other medical professional (eg, anaesthetist or nurse) who is not reported to have received training in geriatric medicine will be excluded.

The perioperative period will be defined as any time between the 'decision to offer surgery, through to the weeks and months after the procedure'. ${ }^{13}$ Any CGA reported outside of this period will be excluded.

\section{Study records}

\section{Data management}

Citation management and data collection will be undertaken in Covidence. ${ }^{14}$

\section{Selection process}

Title and abstracts from all citations identified in the searches will be screened independently for eligibility by two reviewers (RLM, JDB). Screening of full texts will then be undertaken by the same two reviewers. Discrepancies or disagreements in eligibility will be resolved by a third reviewer.

\section{Data collection process}

Data will be extracted independently by two reviewers using a predefined template developed by the study team. 
Any discrepancies or disagreements in data extraction will be resolved by a third reviewer.

\section{Data items}

Data items collected include:

1. Participants: sex, age, number randomised, target sample size, reasons for non-recruitment, surgical specialty, emergency/elective population.

2. Interventions: description of interventions including: components of CGA, healthcare professional delivering intervention, assessment/management tools used (if relevant), time point delivered, duration of time spent with patient, detail of assessment made, detail of care delivered, setting of intervention (eg, clinic, separate ward).

3. Standard care: comparator description, healthcare professionals delivering care in comparator/control group.

4. Outcomes: list of reported outcomes, quantitative data for 11 key areas as defined by COMPAC-StEP working group where possible, including patient comfort, clinical indicators, cognition and stroke, cardiovascular, respiratory, renal, bleeding, morbidity, survival, patient centred outcomes and healthcare resource utilisation. $^{12}$

\section{Risk of bias}

Risk of bias at the outcome level for primary outcomes only will be assessed using the Cochrane risk of bias tool, V.2. ${ }^{15}$

\section{Data synthesis}

A narrative synthesis will be presented for all qualitative outcomes. Content analysis will result in detail of the intervention, assessments and outcomes presented in tabulated form, summarising each study side by side as adapted from similar studies. ${ }^{16}{ }^{17}$ The objectives will be organised according to the definition and domains described in a 1987 conference consensus paper, supplemented with definitions and domains extracted through an iterative process from immersion in the literature. ${ }^{9}$

No meta-analysis will be undertaken as the primary aim of this review is to describe the CGA intervention within each of the trial settings. A simple summary of reported statistics in each trial will be presented.

\section{Patient and public involvement}

There was patient and public involvement in the development of this research question and design of the study via the geriatric perioperative care team at North Bristol National Health Service (NHS) Trust. A formal focus group will be held before publication of the final review.

\section{ETHICS AND DISSEMINATION}

No ethical approval is required for systematic reviews. The study will be disseminated through peer-reviewed manuscript published in a journal and presentation at conferences.
Twitter Rachael Lucia Miller @rachaellucia

Contributors RLM and JDB are joint first authors and PB and RH are joint final authors. RLM, JDB, RM, PB and RH have contributed fully to the concept and design of the review for which this protocol has been written. All authors also all contributed to the writing of this protocol and reviewed the manuscript before submission. RLM wrote the literature search. No data collection or analysis has occurred for this protocol. $\mathrm{RH}$ is the guarantor of the review.

Funding This study was funded by Enid Linder and infrastructure support from the Royal College of Surgeons of England Bristol Surgical Trials Centre. This study was supported by the NIHR Biomedical Research Centre at University Hospitals Bristol and Weston NHS Foundation Trust and the University of Bristol. RLM is an NIHR Academic clinical fellow (ACF-2019-25-005). PB is supported by Research Capability Funding from the Research and Innovation, North Bristol NHS Trust.

Disclaimer The views expressed in this publication are those of the author(s) and not necessarily those of the NHS, the National Institute for Health Research, the Department of Health and Social Care.

Competing interests None declared.

Patient consent for publication Not applicable.

Provenance and peer review Not commissioned; externally peer reviewed.

Supplemental material This content has been supplied by the author(s). It has not been vetted by BMJ Publishing Group Limited (BMJ) and may not have been peer-reviewed. Any opinions or recommendations discussed are solely those of the author(s) and are not endorsed by BMJ. BMJ disclaims all liability and responsibility arising from any reliance placed on the content. Where the content includes any translated material, BMJ does not warrant the accuracy and reliability of the translations (including but not limited to local regulations, clinical guidelines, terminology, drug names and drug dosages), and is not responsible for any error and/or omissions arising from translation and adaptation or otherwise.

Open access This is an open access article distributed in accordance with the Creative Commons Attribution 4.0 Unported (CC BY 4.0) license, which permits others to copy, redistribute, remix, transform and build upon this work for any purpose, provided the original work is properly cited, a link to the licence is given, and indication of whether changes were made. See: https://creativecommons.org/ licenses/by/4.0/.

ORCID iD

Rachael Lucia Miller http://orcid.org/0000-0001-7918-4196

\section{REFERENCES}

1 NELA Project Team. Sixth patient report of the National emergency laparotomy audit rcoA London, 2020. Available: https://www.nela. org.uk/reports [Accessed 19th Nov 2020].

2 Royal College of Surgeons. Access all ages: assessing the impact of ages on access to surgical treatment. Available: https://www.rcseng. ac.uk/library-and-publications/rcs-publications/docs/access-allages/ [Accessed 14 Jan 2021].

3 Aitken RM, Partridge JSL, Oliver CM, et al. Older patients undergoing emergency laparotomy: observations from the National emergency laparotomy audit (NELA) years 1-4. Age Ageing 2020;49:656-63.

4 Gardner M, Shepperd S, Godfrey M, et al. Comprehensive geriatric assessment in hospital and hospital-at-home settings: a mixedmethods study. Health Serv Deliv Res 2019;7:1-206.

5 Ellis G, Gardner M, Tsiachristas A, et al. Comprehensive geriatric assessment for older adults admitted to hospital. Cochrane Database Syst Rev 2017;9:CD006211.

6 Eamer G, Taheri A, Chen SS, et al. Comprehensive geriatric assessment for older people admitted to a surgical service. Cochrane Database Syst Rev 2018;1:CD012485.

7 National Institute of Health and Care Excellence. Quality statement 2: comprehensive geriatric assessment | transition between inpatient hospital settings and community or care home settings for adults with social care needs | quality Standards | NICE. Available: https:// www.nice.org.uk/guidance/QS136/chapter/Quality-statement-2Comprehensive-geriatric-assessment [Accessed 24 Nov 2020].

8 Parker SG, McCue P, Phelps K, et al. What is comprehensive geriatric assessment (CgA)? an umbrella review. Age Ageing 2018;47:149-55.

9 National Institutes of Health. The National Institutes of health $(\mathrm{NIH})$ consensus development program: geriatric assessment methods for clinical decision making. Available: https://consensus.nih.gov/1987/ 1987GeriatricAssessment065html.htm [Accessed 24th Nov 2020]. 
10 Craig P, Dieppe P, Macintyre S. Developing and evaluating complex interventions: new guidance. UK medical Research Council, 2008. Available: www.mrc.ac.uk/complexinterventionsguidance [Accessed 26th Nov 2020].

11 Moher D, Shamseer L, Clarke M. Preferred reporting items for systematic review and meta-analysis protocols (PRISMA-P) 2015 statement. Rev Esp Nutr Humana y Diet 2016;20:148-60.

12 Myles PS, Grocott MPW, Boney O, et al. Standardizing end points in perioperative trials: towards a core and extended outcome set. $\mathrm{Br} \mathrm{J}$ Anaesth 2016;116:586-9.

13 The Royal College of Anaesthetists. Perioperative medicine: the pathway to better surgical care. Available: https://cpoc.org.uk/sites/
cpoc/files/documents/2019-11/RCOA Perioperative Vision Document 2014.pdf [Accessed 18th Dec 2020].

14 Covidence. Covidence - Better systematic review management. Available: https://www.covidence.org/ [Accessed 7th Sept 2020].

15 Higgins JPT, Altman DG, Gøtzsche PC, et al. The Cochrane collaboration's tool for assessing risk of bias in randomised trials. BMJ 2011;343:d5928.

16 Dixon-Woods M, Agarwal S, Young B. Integrative approaches to qualitative and quantitative evidence, 2004.

17 Gardner M, Shepperd S, Godfrey M. Comprehensive geriatric assessment in hospital and hospital-at-home settings: a mixedmethods study. NIHR Journals Libr 2019. 